\title{
EFECTOS ALELOPÁTICOS DE RESIDUOS DE SORGHUM HALEPENSE (L.) SOBRE DOS ARVENSES DICOTILEDÓNEAS EN CONDICIONES DE LABORATORIO.
}

\section{ALLELOPATHIC EFFECTS OF RESIDUES OF SORGHUM HALEPENSE (L.) ON TWO DICOT WEEDS IN LABORATORY CONDITION}

\author{
Lisette Alonso Sánchez¹, Leónides Castellanos González², Isabel Ortega \\ Meseguer ${ }^{3}$
}

\begin{abstract}
Entidad
${ }^{1}$ Estación Territorial de Protección de Plantas Cumanayagua. etppcumanayagua@gmail.com. ORCID: https://orcid.org/0000-0003-2156-2419

${ }^{2}$ Facultad de Ciencias Agrarias. Universidad de Cienfuegos. Carretera a Rodas km 3. Cienfuegos. Iclcastell@gmail.com. https://orcid.org/0000-0001-9285-4879

${ }^{3}$ Laboratorio Provincial de Sanidad Vegetal Cienfuegos. Carretera de Palmira Km 4. ybarmax@mpcfg.co.cu. https://orcid.org/0000-0003-4263-8056
\end{abstract}

\section{Resumen}

La investigación tuvo como objetivo evaluar el efecto alelopático de un extracto acuoso de Sorghum halepense (L.) Pers sobre dos arvenses dicotiledóneas Amaranthus dubius Mart. y de Euphorbia heterophylla L. en pre y post emergencia. Se desarrollaron cuatro ensayos, dos con cada especie de arvense desde febrero 2015 hasta agosto de 2016 en el Laboratorio Provincial de Sanidad Vegetal Cienfuegos (LAPROSAV). Los tratamientos empleados fueron de 0, 40, 60,80 y $100 \mathrm{~g}$ de rizomas fraccionados de la planta $S$. halepense. en $2 \mathrm{~kg}$ de suelo. Los ensayos se dispusieron en un diseño completamente aleatorizado $5 \times 5$ (cinco tratamientos y cinco repeticiones). Las unidades experimentales estuvieron constituidas por magentas donde se ubicaron 20 semillas de las arvenses. A los 12 días se evaluó el porcentaje de germinación o supervivencia, la longitud de la radícula y del hipocótilo. Con esta información se realizaron los análisis de varianza. Las medias se compararon con la prueba de Tukey P $\leq 0,05$, Se usó el paquete SPSS para Windows versión 15. Los residuos de Sorghum halepense (L.) Pers. ocasionaron efecto inhibitorio en la germinación y sobrevivencia de Euphorbia heterophylla L., en pre y post emergencia, redujeron de la radícula en post emergencia y estimularon el hipocótilo en pre emergencia, mientras que sobre $A$. dubius manifestaron acción inhibitoria en el porcentaje de germinación y sobrevivencia. y disminuyeron la longitud del hipocótilo tanto en pre como en post emergencia y afectaron la radícula a la dosis más alta en pre emergencia.

Palabras clave:

Alelopatía, fitotoxicidad, arvenses, germinación, radícula, hipocótilo

\section{Abstract}

The research aimed to evaluate the allelopathic effect of an aqueous extract of Sorghum halepense (L.) Pers on two dicot weeds Amaranthus dubius Mart. and of Euphorbia heterophylla L. in pre and post emergence. Four trials were carried out, two with each weed species from February 2015 to August 2016 at the Cienfuegos Provincial Plant Health Laboratory (LAPROSAV). The treatments used were $0,40,60,80$ and $100 \mathrm{~g}$ of fractionated rhizomes from the $S$. halepense plant. in $2 \mathrm{~kg}$ of soil. The trials were arranged in a completely randomized $5 \times 5$ 
design (five treatments and five replications). The experimental units were made up of magentas where 20 weed seeds were located. After 12 days, the percentage of germination or survival, the length of the radicle and the hypocotyl were evaluated. Analysis of variance was performed with this information. The means were compared with the Tukey test $P \leq 0.05$. The SPSS package for Windows version 15 was used. The residues of Sorghum halepense (L.) Pers. caused an inhibitory effect on the germination and survival of Euphorbia heterophylla L., in pre and post emergence, they reduced the radicle in post emergence and stimulated the hypocotyl in pre emergence, while on $A$. dubius they showed inhibitory action on the percentage of germination and survival. and they decreased the hypocotyl length both in pre and post emergence and affected the radicle at the highest dose in pre emergence.

Key words:

Allelopathy, phytotoxicity, weeds, germination, radicle, hypocotyl

\section{INTRODUCCIÓN}

Las arvenses pueden llegar a producir pérdidas significativas en los cultivos, estimándose entre $40-45 \%$ en vegetales de hojas, $35 \%$ en tomate, $25-30 \%$ en berenjena, $15-25 \%$ en coliflor, $15-20 \%$ cucurbitáceas. Se llega a invertir más del $40 \%$ del tiempo laboral, fundamentalmente a través del desyerbe (Singh et al., 2014) y en la mayoría delos casos se recurre a la aplicación de herbicidas químicos.

Una de las principales críticas a la agricultura de conservación es su elevada dependencia a los herbicidas en comparación con los sistemas con labranza. Adicionalmente, en muchas áreas enfocadas en la agricultura de conservación, los herbicidas no están disponibles o tienen precios inaccesibles, por lo que el control de malezas debe realizarse por otros medios (Nichols et al., 2015; Melo et al., 2017).

Cada vez más es necesario la implementación de una agricultura sostenible, este tipo de producción demanda controles alternativos que impacten menos al medio ambiente y a las personas, se pueden enumerar como ejemplos los extractos y residuos de plantas, aceites y mezclas de plantas, que son productos viables (Moreno y Rueda, 2016; Santos, 2018).

El término alelopatía se refiere a los efectos perjudiciales 0 benéficos que como resultado de la acción de compuestos químicos liberados por una planta, ejercen su acción en otra. Siguiendo esta definición en todo fenómeno alelopático existe una planta donadora que libera al medio ambiente por una determinada vía compuestos químicos los cuales al ser incorporados por otra planta (receptora) provocan un efecto perjudicial o benéfico sobre germinación, crecimiento o desarrollo de esta última (Sampietro, 2005).

Se refieren en la literatura productos naturales donde los aleloquímicos de plantas, así como algunos derivados sintéticos, tienen probada actividad herbicida, como es el caso de sorgoleona (Sorghum bicolor), artemisina (Artemisia annua), leptospermone (Leptospermum scoparium), sarmentina (Piper longum); todos con aplicaciones diversas incluidas la síntesis y las mezclas con otros herbicidas comerciales (Dayan et al., 2015).

El Don Carlos (Sorghum halepense L. Pers.) es una de las principales malezas invasoras perennes a nivel mundial que ocasiona serios inconvenientes en el 
crecimiento y desarrollo de los cultivos. La infestación por esta arvense en las áreas agrícolas constituye un grave problema debido a las dificultades para su control. La misma presenta una elevada tasa de producción de rizomas. Una planta puede generar de 40 a $90 \mathrm{~m}$ de rizomas por estación de crecimiento y 28.000 semillas por planta (Horowitz, 1973; Gutierrez et al., 2016).

Se ha demostrado el efecto adverso de las sustancias alelopáticas presentes en $S$. halepense sobre sobre la biomasa aérea y radical de plántulas de trigo y se han dado recomendaciones para continuar las investigaciones sobre este efecto en condiciones de campo (Acciaresi y Asenjo, 2003). Samprieto (2005) menciona también a $S$ halepense dentro de las plantas con potencial alelopático sobre algunos cultivos.

Aunque recientemente se informó sobre el efecto alelopático de un extracto acuoso Sorghum halepense sobre dos dicotiledóneas Euphorbia heterophylla L. y Amaranthus dubius Mart (Alonso et al., 2017; Angulo et al., 2017) todavía es escasa la información del efecto que realizan los residuos de los rizomas de Don Carlos que quedan en el suelo, sobre estas y otras arvenses.

Teniendo en consideración estos antecedentes el objetivo de este trabajo fue evaluar el efecto alelopático de fragmentos de los rizomas de Sorghum halepense (L.) Pers sobre Euphorbia heterophylla L. y Amaranthus dubius Mart.

\section{METODOLOGIA}

La investigación se desarrolló desde febrero 2015 hasta agosto 2016 en el Laboratorio Provincial de Sanidad Vegetal Cienfuegos (LAPROSAV) con la finalidad de determinar el efecto alelopático de partes de las plantas de Sorghum halepense (L.) Pers. sobre dos arvenses dicotiledóneas en pre y post emergencia.

Se seleccionaron como plantas a controlar las arvenses Amaranthus dubius Mart. (bledo) y Euphorbia heterophylla L. (hierba lechosa) ya que se consideran invasoras en el territorio de Cienfuegos, Cuba. Las plantas de las dos especies se recolectaron en la fase de fructificación en el Organopónico Empresa Municipal Alimentaria de Cumanayagua (EMA II) en diciembre del 2015, se colocaron en bolsas de plástico y se trasladaron al LAPROSAV Cienfuegos para su identificación y procesamiento, diagnosticar el porcentaje de germinación de las semillas y certificarlas como libres de agentes nocivos.

Las semillas de las arvenses fueron desprovistas de sus vainas de forma manual, libres de impurezas y en buen estado físico, almacenándolas en recipientes cerrados y a temperatura de $13^{\circ} \mathrm{C}$.

Los ejemplares de Sorghum halepense se obtuvieron en áreas de la Unidad Empresarial de Base (UEB) Santa Martina, perteneciente a la Empresa Pecuaria Sierrita, del municipio Cumanayagua, provincia Cienfuegos. Las plantas se tomaron en la fase de inflorescencia y trasladadas para su identificación a la Sección de Herbología del LAPROSAV Cienfuegos. Una vez confirmado su diagnóstico se lavaron los rizomas con agua corriente.

Para todos ensayos se utilizó un suelo aluvial que se obtuvo en la Unidad Empresarial de Base (UEB) Santa Martina, perteneciente a la Empresa Pecuaria Sierrita. Este se esterilizó a temperatura de $140^{\circ} \mathrm{C}$ durante una hora en estufa marca Membert.

Para determinar el efecto alelopático de los residuos de $S$. halepense sobre las dos dicotiledóneas se realizaron cuatro 
ensayos, dos en tratamientos pre emergentes y dos en tratamientos post emergentes, uno sobre cada arvense en específico.

Para los ensayos de los tratamientos pre emergente la preparación de los fragmentos de las plantas se llevó a cabo según lo sugerido por Sampietro (2005) realizando cortes de los rizomas en pequeños trozos de $0,5 \mathrm{~cm}$. Los tratamientos empleados fueron de 0,40 , 60,80 y $100 \mathrm{~g}$ de rizomas fraccionados de la planta $S$. halepense. en $2 \mathrm{~kg}$ de suelo. Se esperó 24 horas para efectuar la siembra de las semillas de las arvenses en estos los ensayos.

Los tratamientos se dispusieron en un diseño completamente aleatorizado $5 \times 5$ (cinco tratamientos y cinco repeticiones). Las unidades experimentales (repeticiones) estuvieron constituidas por las magentas plásticas las cuales tenían de 110 milímetros de diámetro inferior, 120 milímetros de diámetro superior y una altura de 60 milímetros. En cada magenta fueron colocados $250 \mathrm{~g}$ del suelo con los residuos de las plantas.

En cada magenta se sembraron 20 semillas de la arvense según los tratamientos a evaluar, las cuales se regaron con agua destilada estéril diariamente, hasta $60 \%$ de capacidad de campo.

Los pesajes de suelo y rizomas de las plantas se realizaron en una balanza mecánica, marca OHAUS, verificada por la Oficia Territorial de Normalización de Cienfuegos.

Las magentas se incubaron en cámara de germinación a una temperatura de $30.0 \pm 0.1^{\circ} \mathrm{C}$ durante 16 horas de luz y $25.0 \pm 0.1^{\circ} \mathrm{C}$ durante ocho horas para todas las concentraciones que se ensayaron y el testigo.
Para evaluar el efecto alelopático post emergente se añadió a cada unidad experimental $250 \mathrm{~g}$ el suelo estéril y se sembraron 20 semillas de cada especie de arvense por magenta, las cuales se regaron con agua destilada estéril diariamente, hasta $60 \%$ de capacidad de campo.

Al quinto día de la siembra cuando se observaron un $50 \%$ de las semillas germinadas en las magentas, se aplicaron proporcionalmente con la ayuda de una pinza los residuos de $S$ halepense según la cantidad establecida para el tratamiento pre emergente, o sea, 0, 40, 60, 80 y $100 \mathrm{~g}$ de rizomas fraccionados de la planta de $S$. halepense por cada $2 \mathrm{~kg}$ de suelo. Se tuvo cuidado de que las facciones de rizomas estuvieran bien en contacto con el suelo y no estuvieran en contacto con las pequeñas plántulas.

En los ensayos con tratamientos pre emergente se evaluaron cada tres días las siguientes variables: porcentaje de germinación, longitud de la radícula y longitud del hipocótilo; y para el efecto post emergente las variables evaluadas fueron: porcentaje de sobrevivencia de las plántulas, longitud de la radícula y longitud del hipocótilo. Las mediciones se realizaron con auxilio de una regla milimetrada.

Se tomó la información del día 12 después de aplicado los residuos para realizar los análisis estadísticos, tiempo de incubación necesario para cuantificar el número de semillas germinadas y las plántulas sobrevivientes.

Los resultados fueron sometidos a un análisis de varianza previa comprobación de la normalidad de los datos de cada variable en cada ensayo por la prueba de Komodorov- Smirnov. Los datos en porcentajes se transformaron en 
arcsen $\sqrt{ } \% / 100$. Las medias se compararon con la prueba de Tukey $P \leq 0,05$. Se usó el paquete SPSS para Windows versión 21 (IBM, 2012).

\section{ANALISIS $Y$ DISCUSIÓN DE RESULTADOS}

Se verificó efecto alelopático de los residuos de los rizomas de $S$. halepense a todas las dosis estudiadas contra Euphorbia heterophylla en pre emergencia ya que se observó efecto inhibitorio en la germinación. Los mayores efectos se observaron a las dosis de 80 y 100 gramos $/ 2 \mathrm{~kg}$ de suelo, que difirieron significativamente de las demás dosis y el testigo. Los porcentajes en estos tratamientos fueron inferiores al testigo en más de un $60 \%$. (Tabla 1 ).

Tabla 1. Efecto de fracciones de rizomas de Sorghum halepense contra Euphorbia heterophylla en pre emergencia

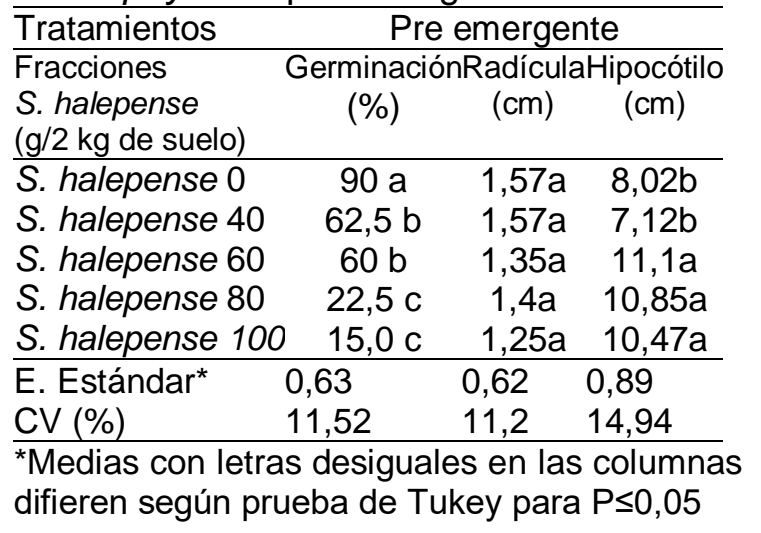

Estos resultados se ratifican los que obtuvieron Blanco et al. (2007), quienes plantearon que Sorghum halepense produce exudados radicales inhibitorios para la germinación de especies de plantas no cultivadas y malezas.

Al evaluar la longitud de la radícula se observó que no hubo diferencia estadística entre los tratamientos y el testigo, por lo que no hubo efecto en pre emergencia a ninguna de las dosis empleadas de las fracciones de $S$. halepense.
Las fracciones de $S$. halepense incorporadas al suelo produjeron una estimulación del hipocótilo a las dosis de 60,80 y 100 gramos, con diferencia estadística de la de 40 gramos y del testigo. No se encontró resultados en la literatura sobre el efecto estimulador del hipocótilo de otras especies causados por exudados de $S$. halepense, sin embargo, también se han encontrado otros residuos de plantas con efectos estimulantes en el rendimiento agrícola, tal es el caso del empleo de residuos de Jatropha curcas (0,75-3 tha- 1 ), el cual incrementó el rendimiento en mijo perla (Pennisetum glaucum (L.) R.Br.), en col (Brassica oleracea var. capitata L.) y en arroz (Oryza sativa L.) (Dubey et al., 2015).

Con respecto al efecto de Sorghum halepense en fracciones de la planta contra Euphorbia heterophylla en post emergencia, se observó inhibición de la sobrevivencia a todas las dosis estudiadas, aunque superiores para las dosis de 60, 80 y $100 \mathrm{~g}$ que difirieron estadísticamente de 40 gramos y el testigo (Tabla 2).

Tabla 2. Efecto de fracciones de rizomas de Sorghum halepense contra Euphorbia heterophylla en post emergencia

\begin{tabular}{|c|c|c|c|}
\hline Tratamientos & Post emerge & ente & \\
\hline $\begin{array}{l}\text { Fracciones } \\
\text { S. halepense } \\
\text { (g/2 kg de } \\
\text { suelo) }\end{array}$ & $\begin{array}{l}\text { Porcentaje } \\
\text { De sobre. } \\
\text { (\%) }\end{array}$ & $\begin{array}{l}\text { Radícular } \\
\text { (cm) }\end{array}$ & $\begin{array}{l}\text { lipocótilo } \\
\text { (cm) }\end{array}$ \\
\hline S. halepense 0 & $90 a$ & $1,57 a$ & $8,02 a$ \\
\hline S. halepense 40 & $62,5 \mathrm{~b}$ & $1,47 a b$ & $7,97 a$ \\
\hline S. halepense 60 & $37,5 \mathrm{c}$ & $1,65 a$ & $9,07 a$ \\
\hline S. halepense 80 & $40 \mathrm{bc}$ & $1,22 a b$ & $8,15 a$ \\
\hline $\begin{array}{l}\text { S. halepense } \\
100\end{array}$ & $32,5 \mathrm{c}$ & $1,1 \mathrm{~b}$ & $8,75 a$ \\
\hline E. Estándar* & 0,64 & 0,62 & 0,87 \\
\hline CV (\%) & 12,21 & 11,1 & 13,75 \\
\hline
\end{tabular}

${ }^{*}$ Medias con letras desiguales en las columnas difieren según prueba de Tukey para $\mathrm{P} \leq 0,05$

La longitud de la radícula sólo fue reducida significativamente por la dosis más alta de las fracciones de $S$. halepense $(100 \mathrm{~g} / 2 \mathrm{~kg}$ de suelo). El resto de los tratamientos no 
difirieron del testigo, aunque los de 40 y 80 $\mathrm{g}$ de no difirieron del de la dosis más alta.

Efectos similares sobre la longitud de la radícula se reportan con Ipomoea carnea Jacq, donde los extractos acuosos de las hojas ( $1-5 \% \mathrm{p} / \mathrm{v})$ logran inhibir la longitud de la radícula, actividad que aumentó con la concentración (Joshi et al., 2015).

No hubo efecto de los tratamientos sobre la longitud del hipocótilo con respecto al testigo, lo que pudiera tener su explicación porque ya había un porcentaje de las plantas germinadas.

Al analizar el efecto de Sorghum halepense en fracciones de la planta contra Amaranthus dubius en pre emergencia se observó que todos los tratamientos difirieron estadísticamente con relación al testigo, por lo que hubo acción sobre la germinación a todas las dosis aplicadas, aunque en la de 80 y $100 \mathrm{~g}$ fue superior al diferir del testigo y de las dosis de 40 y 60 gramos. A las dosis más altas, 80 y $100 \mathrm{~g}$, se redujo en más del $80 \%$ la germinación respecto al testigo (Tabla 3 ).

Tabla 3. Efecto de fracciones de rizomas de Sorghum halepense contra Amaranthus dubius en pre emergencia

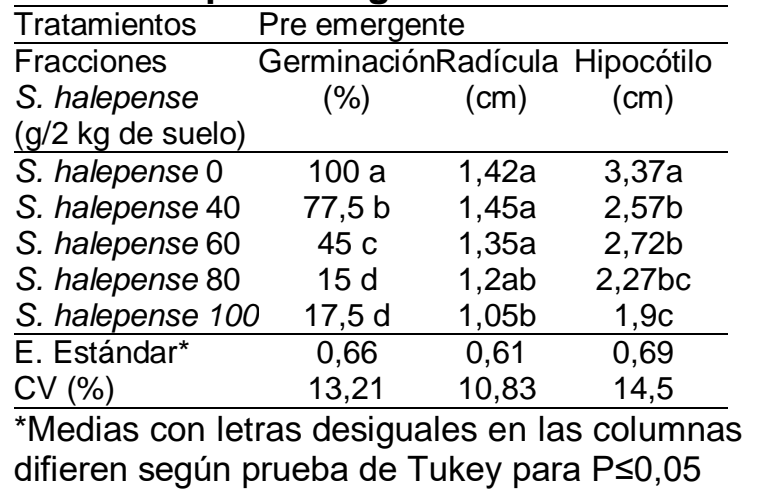

Sobre la radícula sólo hubo acción a la dosis máxima, que difirió estadísticamente de las demás y con el testigo, mientras que sobre el hipocótilo a las dosis de 80 y 100 $\mathrm{g}$ fue mayor el efecto al diferir estadísticamente del resto de los tratamientos y del testigo.

Las fracciones de rizomas de $S$. halepense tuvieron mayor efecto sobre la germinación de $A$. dubius a las dosis de 80 y $100 \mathrm{~g}$, resultados se asemejan a los de estudios realizados por Acciaresis y Asenjo (2003) que informan que con esta poacea se afectó el poder germinativo de las semillas de maíz y frijol.

El efecto sobre el hipocótilo de $A$. dubius a las dosis de 80 y $100 \mathrm{~g}$ se corresponde con los resultados de Nicollie et al. (1983), quienes encontraron que los extractos y residuos de los rizomas de $S$. halepense mostraron efecto negativo sobre el crecimiento de la Soya.

Los re4siduos de Sorghum halepense aplicados en post emergencia contra Amaranthus dubius manifestaron efecto sobre la sobrevivencia de las plantas a todas las dosis, aunque superiores para las dosis de 60, 80 y 100 gramos que difirieron de la dosis de 40 gramos y del testigo (Tabla 4).

Tabla 4. Efecto de fracciones de rizomas de Sorghum halepense contra Amaranthus dubius en post emergencia

\begin{tabular}{|c|c|c|c|}
\hline Tratamientos & Post emerge & nte & \\
\hline $\begin{array}{l}\text { Fracciones } \\
\text { S. halepense } \\
\text { (g/2 kg de suelo) }\end{array}$ & $\begin{array}{c}\text { Porcentaje } \\
\text { Sobreviven. } \\
(\%)\end{array}$ & $\begin{array}{l}\text { Radícula } \\
(\mathrm{cm})\end{array}$ & $\begin{array}{l}\text { Hipocótilo } \\
\text { (cm) }\end{array}$ \\
\hline S. halepense 0 & $100 \mathrm{a}$ & $1,42 a$ & $3,37 a$ \\
\hline S. halepense 40 & $80 \mathrm{~b}$ & $1,4 a$ & $3,05 a b$ \\
\hline S. halepense 60 & $37,5 c$ & $1,27 a$ & $2,42 b c$ \\
\hline S. halepense 80 & $27,5 c$ & $1,25 a$ & $1,92 c$ \\
\hline S. halepense 100 & 20 & $1,27 a$ & $2,27 c$ \\
\hline E. Estándar* & 0,67 & 0,61 & 0,68 \\
\hline CV (\%) & 13,86 & 10,95 & 14,47 \\
\hline
\end{tabular}

No hubo efecto sobre la longitud de la radícula de $A$. dubius tratadas con fragmentos de $S$. halepense en post emergencia como había ocurrido sobre $E$ heterophylla, sin embargo, hubo efecto alelopático sobre el hipocótilo, ya que los 
tratamientos a las dosis de 60,80 y $100 \mathrm{~g}$ redujeron significativamente la longitud con relación al testigo. El tratamiento a la dosis de $40 \mathrm{~g}$ no difirió del testigo ni del de la dosis de $60 \mathrm{~g}$, lo que también se corresponde con lo observado en soya por Nicollie et al. (1983).

Los rizomas fraccionados de $S$. halepense mostraron resultados similares contra $E$. heterophylla tanto en pre como en post emergencia ya que hubo un efecto inhibitorio en la sobrevivencia de las plántulas. Sobre la longitud de la radícula no hubo acción en pre emergencia, mientras que en post emergencia a la dosis más alta difirió del testigo. Estos dos experimentos también difirieron en cuanto a la longitud del hipocótilo, pues hubo una estimulación en pre emergencia, y en post emergencia no hubo diferencia entre los tratamientos.

En relación al porcentaje de sobrevivencia de las plantas tratadas con residios vegetales Hernández (2017) comprobó que los residuos de Phyla strigulosa (M.Mart. \& Gal.) Mold., Sphagneticola trilobata L. Pruski e Ipomoea batatas (L.) Lam, afectaron el porcentaje de germinación de plántulas de varias arvenses como Portulaca oleracea L. Chamaesyce sp. y Amaranthus sp. en la medida que se aumentaba la dosis, de forma similar a lo que se observó en el presente estudio al evaluar el efecto alelopático de $S$. halepense sobre las plántulas de $A$. dubius y E. heterophylla.

No se observó un efecto similar sobre la longitud de la radícula de $A$. dubius y $E$ heterophylla tratadas con fragmentos de $S$. halepense en los tratamientos pre y post emergentes lo que demuestra la dependencia de la alelopatía de la cantidad de residuo y la sensibilidad de la especie de planta que recibe el efecto alelopático; lo que ha sido señalado también por Muhammad y Majeed (2014), al evaluar los efectos de los extractos acuosos de Helianthus annuus L. sobre maíz.

Samprieto (2005) señala que los efectos de inhibición de la germinación de las plantas receptoras de sustancias alelopáticas pueden deberse a cierta limitación en la disponibilidad de enzimas hidrolíticas, AIA y reducción de la mitosis.

También en la literatura se informa que las raíces de las plantas de Mentha $x$ piperita L. desarrolladas a diferentes concentraciones de un extracto acuoso de Sorghum halepense mostraron daño significativo de las membranas celulares por efecto de los extractos de la arvense con respecto a las no expuestas (Arango et al., 2013), lo que podría ser una de las causas de efecto sobre el crecimiento de la radícula sobre las dos especies de arvenses estudiadas, aunque diferentes autores describen otros sitios de acción de los aleloquímicos, que afectan las funciones de enzimas específicas (Singh et al., 2014; Mondal et al., 2015).

Entre los compuestos químicos de acción alelopática identificados a partir de la descomposición de residuos de $S$. halepense se encuentran las $p$ benzoquinonas (sorgoleona), glucósidos cianogenéticos, taninos y ácidos fenólicos (p-cumárico, ferúlico, vanílico) (Sene et al., 2001), cuestión a tener en cuenta en los efectos encontrados por los fragmentos de esta planta sobre $A$. dubius y $E$ heterophylla en el presente trabajo y también por Alonso et al. (2017) cuando usó extractos acuosos de Don Carlos contra estas dos especies de arvenses.

Los presentes resultados son alentadores para la reducción las poblaciones de las arvenses en estudio sobre todo por el efecto post y pre emergente sobre la germinación y la sobrevivencia. Estos son comparables con los de Hernández (2016) quien observó efecto inhibidor de los residuos de $P$. strigulosa, $S$. trilobata, e $I$. 
batatas sobre la germinación de varias arvenses dependiendo de la dosis y la sensibilidad de la especie receptora, lo que sugiere continuar las investigaciones en condiciones de campo para precisar cómo se pueden introducir estos resultados para el manejo de $A$. dubius y $E$ heterophylla en áreas pequeñas como huertos organopónicos, donde puedan obtenerse con facilidad en áreas aledañas rizomas de S. halepense

\section{CONCLUSIONES}

Los residuos de Sorghum halepense (L.) Pers. ocasionaron efecto inhibitorio in "vitro" sobre la germinación y sobrevivencia de Euphorbia heterophylla L., en pre y post emergencia y produjeron una reducción de la radícula en post emergencia y una estimulación del hipocótilo aplicados en pre emergencia, sin embargo. contra Amaranthus dubius Mart manifestaron acción inhibitoria en el porcentaje de germinación y sobrevivencia, disminuyeron la longitud del hipocótilo tanto en pre como en post emergencia y afectaron la radícula a la dosis más alta en pre emergencia, lo que sugiere continuar los estudios en condiciones de campo.

\section{Referencias Bibliográficas}

Acciaresi, H., Asenjo, C. (3003). Efecto alelopático de Sorghum halepense (L.) Pers. sobre el crecimiento de la plántula y la biomasa aérea y radical de Triticum aestivum (L.). Ecología Austral, 13: 49-61, 2003.

Alonso, L., Castellanos, L., Ortega, I., Martínez, E. (2017). Efecto alelopático de un extracto acuoso de Sorghum halepense (L.) Pers. sobre dos dicotiledóneas. Revista Agroecosistemas, 5(2): 25-31, 2017.

Angulo, W. J., Mendoza, J. A. y Uriel, H. U. (2017). Análisis de la vulnerabilidad por fenómenos de remoción en masa en la Cuenca Tanauca estudio de caso. Revista Ambiental Agua, Aire y Suelo. ISSN 1900-
9178. Volumen (8), Numero (2). DOI: https://doi.org/10.24054/19009178.v2 .n2.2017.3276

Arango, C., Ruscitti, M., Ronco, M., Beltrano, J. (2013). Influencia de los extractos acuosos de rizomas de sorgo de Alepo (Sorghum halepense L.) sobre la micorrización y el crecimiento de plantas de Mentha $x$ piperita L. Horticultura Argentina 32(78): 22-29.

Blanco, Y., Hernández, I., Urra, I., Leyva, A. (2007). Potencial alelopático de diferentes extractos de girasol (Helianthus annus, L), maíz (Zea mays L.), frijol (Phaseolus vulgaris, L.) y boniato (Ipomoea batata, L.) sobre el crecimiento y desarrollo inicial del frijol común (Phaseolus vulgaris, L), Cultivos Tropicales. 28(3): 5-9,.

Dayan, F.E., Owens, D.K., Watson, S.B., Asolkar, R., Boddy, L. Sarmentine. (2015). A natural herbicide from Piper species with multiple herbicide mechanisms of action. Frontiers in Plant Science, 6: 222-259,

Dubey, G., Bharati, K., Ahirwar, U., Mishra, R., Tiwari, S., Mohanty, S.R. (2015). Prospect of bioactive molecules from Jatropha curcas to improve soil and microbial quality for sustainable agriculture. African Journal of Microbiology Research, 9(3), 140-146, 2015.

Gutierrez, T., Castellanos, C. y Hernández, N. (2016). El ordenamiento territorial frente a las consecuencias de los cambios climáticos. Revista Ambiental Agua, Aire y Suelo. ISSN 1900-9178. Volumen (7), Numero

DOI: https://doi.org/10.24054/19009178.v2 .n2.2016.3338

Hernández, M. (2016). Potencial alelopático de Phyla strigulosa (M.Mart. \& Gal.) Mold., Sphagneticola trilobata L. Pruski e Ipomoea batatas (L.) Lam sobre arvenses y cultivos. Tesis presentada en opción al grado científico de Doctor en Ciencias Agrícolas. Universidad Central de 
Las

Villas.

DOI:

10.13140/RG.2.1.4941.9929.

Horowitz, M. (1973). Spatial Growth of Sorghum halepense. Weed Research, 13:200-208.

IBM SPSS. (2012). Statistics for Windows, Version 21.0. New York: IBM Corp., 2012. ISBN $-13: 978-0205985517$. www.ibm.com/analytics/us/en/technology/s pss/

Joshi, N., Nangia, N., Joshi, A. (2015). Seed germination studies on allelopathic effects of weeds on Vigna radiata L. International Journal of Bioassays, 4(2), 3664-3666

Melo, J., Saavedra, S. y Ramón, J. A. (2017). Evaluación de la adsorcion de $\mathrm{CU}+2$ y azul de metileno en biosorbentes de bajo costo obtenidos a partir de biomasa residual de la agroindustria de cítricos. Revista Ambiental Agua, Aire y Suelo. ISSN 1900-9178. Volumen (8), Numero (2). DOI: https://doi.org/10.24054/19009178.v2 .n2.2017.3277

Mondal, Md.F., Asaduzzaman, Md., Asao, T. (2015). Adverse effects of allelopathy from legume crops and its possible avoidance. American Journal of Plant Sciences, 6(6), 804-810.

Moreno, C. y Rueda, L. (2016). La educación ambiental como herramienta para la recuperación de la cobertura vegetal, mediante prácticas agroecológicas en la comunidad minera asograstorres, asociación de gravilleros de Sabana de Torres. Revista Ambiental Agua, Aire y Suelo. ISSN 1900-9178. Volumen (7), Numero (1). DOI: https://doi.org/10.24054/19009178.v1 .n1.2016.3260

Muhammad, Z., Majeed, A. (2014). Allelopathic effects of aqueous extracts of sunflower on wheat (Triticum aestivum L.) and maize (Zea mays L.). Pakistan Journal of Botany, 46 (5), 1715-1718.
Nichols, V., Verhulst, N., Cox, R., Govaerts, B. (2015). Agricultura de conservación y manejo de malezas. CIMMYT, 9 p.

Nicollie, G., Pope, D., Thompson, A. (1983). Biological activity of dhurrin and other compounds from Johnson grass (Sorghum halepense) J. Agric. Food Chemitry 31: 744-748.

Sampietro, A. (2005). Alelopatía: Concepto, características, metodología de estudio e importancia. Cátedra de Fitoquímica. Instituto de Estudios Vegetales "Dr. Antonio R. Sampietro "Facultad de Bioquímica, Química y Farmacia. Universidad Nacional de Tucumán Ayacucho 461.CP 4000. San Miguel de Tucumán. Argentina.

Sene, M., Gallet, C., Dore, T. (2001). Phenolic compounds in a Sahelian sorghum (Sorghum bicolor) genotype and associated soils. Journal of Chemical Ecology 27:81-92.

Santos P. J. dos. (2018). O efeito alelopático do óleo neem (Azadirachta indica A. Juss) sobre a germinação e crescimento de plantas de alface (Lactuca sativa L.). [Trabalho de conclusão de curso, Universidade Federal da Fronteira Sul] Erechim, Brazil. 2018.

Singh, H.P., Kaur, S., Batish, D.R., Ravinder, K.K. (2014). Ferulic acid impairs rhizogenesis and root growth, and alters associated biochemical changes in mung bean (Vigna radiata) hypocotyls. Journal of Plant Interactions, 9 (1), 267-274. 